\section{Investigación biomédica en Chile: Algunos comentarios}

\section{Reflections on current biomedical research, in Chile}

\section{Sr. Editor:}

Deseo felicitar a los Drs. Valdés, Armas y Reyes por el completo e informativo artículo respecto de las características de la investigación biomédica en Chile'.

Resulta sorprendente percatarse que las compañías farmacéuticas extranjeras invierten tanto como el Estado chileno en investigación biomédica en el país: alrededor de 26 millones de dólares. Esta cifra revela, en primer lugar, la aun menguada participación del Estado en el financiamiento de las ciencias biomédicas en el país. En segundo lugar, al parecer demuestra también que una parte importante, tal vez la mitad, de la agenda científica en este rubro es dirigida por intereses potencialmente ajenos a los problemas biomédicos de Chile. Sorprendente es también, como lo señalan los autores, que el Estado chileno carezca de un registro de acceso público de estas últimas actividades, que permitiría aquilatar los beneficios que ellas proporcionan al país, tanto en la creación de infraestructura, en la formación de personal y en encontrar soluciones para los problemas biomédicos nacionales.

La ausencia de información y de transparencia que produce la falta de un registro de estas actividades se presta también para la generación de problemas éticos que pasan desapercibidos y que potencialmente afectan negativamente los derechos de poblaciones vulnerables en el país, incluyendo niños, ancianos y grupos de bajos niveles educativos y socio económicos ${ }^{2}$. Por ejemplo, ¿qué favor le reporta a la población chilena el ensayo de la efectividad de un antibiótico, droga o vacuna llevado a cabo por una compañía farmacéutica extranjera si una vez comercializada no estará a su alcance por su elevado precio? Similarmente, ¿qué ayuda presta a la solución de un problema de salud pública el ensayo de la efectividad de un antibiótico, droga o vacuna llevado a cabo por una compañía farmacéutica extranjera en la población chilena, si las raíces del problema están en la pobreza y la falta de atención médica o si existen medidas alternativas de prevención y de educación para controlarlos? En ambos casos estos ensayos no debieran llevarse a cabo, ya que tampoco podrían efectuarse en un país industrializado, por la ausencia total de beneficio directo a los sujetos de la experimentacion ${ }^{3,4}$.

Las actividades de investigación de las compañías farmacéuticas en países como Chile muchas veces se llevan a cabo aprovechándose de un relativismo ético que permite vulnerar los derechos humanos de los sujetos de investigación, que debieran ser absolutos ${ }^{3,4}$. Por esta razón, como se señala en el artículo, un país como Chile, con una gran tradición de práctica médica y de salud pública, debiera contar ya con un registro que analice y controle este tipo de investigación. Sin lugar a dudas la Academia Chilena de Medicina y sus miembros, como los autores de este artículo, recogiendo la activa y centenaria tradición de las Academias anglosajonas y europeas, son las llamadas a identificar y proponer soluciones para estos problemas médicos y de derechos humanos. Esperamos que la investigación presentada fructifique con una instancia nacional que registre y haga pública la información discutida.

\section{Dr. Felipe C. Cabello \\ Department of Microbiology and Immunology New York Medical College, Valhalla,} NY, U.S.A.

\section{Referencias}

1. Valdés G, Armas M R, Reyes B H. Principales características de la investigación biomédica actual, en Chile. Rev Med Chile 2012; 140: 484-92.

2. Cabello F. Experimentación en humanos y derechos humanos. ¿Está permitida la experimentación biomédica con niños? Rev Méd Chile 1986; 114: 57-60.

3. Angell M. Ethical Imperialism? Ethics in International collaborative clinical research. N Engl J Med 1990; 319 : 1081-3.

4. Cabello F. Ethical Imperialism? A letter. N Engl J Med 1990; 322: 200.

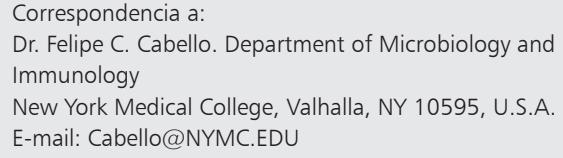

